\title{
Eventeration of the diaphragm with a gastric volvulus
}

\author{
Atulugama N.S, MBBS ${ }^{1}$, Samarajeewa U, MBBS, MD, MRCS ${ }^{2}$, Kollure S.K, \\ MBBS, MS, FRCS(Ed.,Eng.\&Glas.) $)^{3}$ \\ 1 Surgical Registrar, Surgical Unit, National Hospital of Sri Lanka. \\ 2 Senior Surgical Registrar, Surgical Unit, National Hospital of Sri Lanka. \\ 3 Consultant Surgeon, Surgical Unit, National Hospital of Sri Lanka.
}

Key words: Diaphragm; Gastric volvulus.

\section{Introduction}

Eventeration of the diaphragm is defined as an abnormal elevation of the intact diaphragm due to developmental abnormality of the diaphragmatic musculature. This leaves a potential space for herniation of abdominal contents into the thoracic cavity, of which, herniation of the stomach may lead to an intermittent volvulus.

\section{Case report}

A 35 year old man, who presented with epigastric pain and fullness, was retching continuously for one day. There was no active vomiting. He denied alcohol abuse. There was no evidence of upper gastro-intestinal bleeding. On examination, he had epigastric tenderness with localized gaseous distention. Clinically, he was pale and he had a tachycardia. Our initial diagnosis was acute pancreatitis or gastrooesophageal reflux disease.

He had had similar admissions to various hospitals during the last three years and was managed conservatively with proton pump inhibitors and nasogastric tube decompression. Chest x-rays had revealed that he has a dextrocardia. Upper gastrointestinal endoscopy had not been previously undertaken.During the

Correspondnce: S.K. Kollure, Consultant Surgeon, National Hospital of Sri Lanka.

Email: kollure@gmail.com

The Sri Lanka Journal of Surgery 2010; 28(2):22-24 present admission, serum amylase was normal and an erect chest radiograph revealed elevation of the left hemidiaphragm with the cardiac shadow in the right hemi-thorax prompting the suspicion of diaphragmatic eventeration (Figure $1)$.

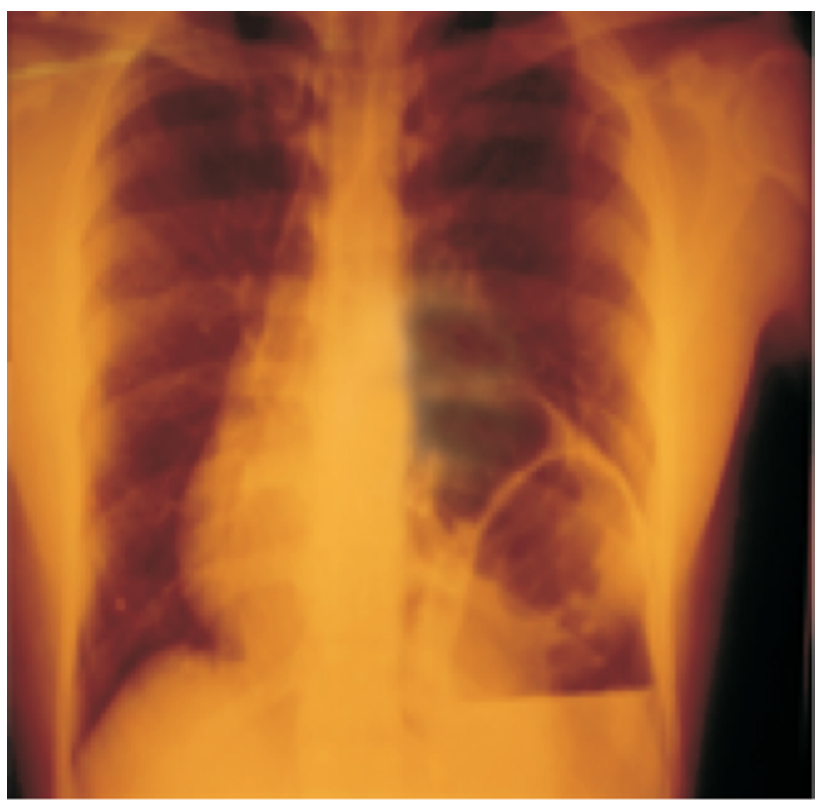

Figure 1. Chest radiograph showing diaphragmatic eventeration

A nasogastric tube was inserted which drained about 2 liters of coffee ground content. Upper endoscopy found a large extra-gastric mass bulging into the posterior wall of the stomach. An exploratory laparotomy was performed following resuscitation. At laparotomy, we found a left sided eventeration of the diaphragm, which consisted of a thin 
muscular membrane with herniation of transverse colon, spleen and the left kidney together with a combined mesentero-axial and organo-axial gastric volvulus. All of the herniating organs were reduced into theabdominal cavity and de-rotation of the volvulus was followed by gastropexy. The diaphragmatic membrane was plicated and reinforced with polypropylene mesh. The patient was discharged five days after operation without complications.

\section{Discussion}

Eventeration of the diaphragm is defined as an abnormal elevation of an intact diaphragm, and most often, is characterized by a developmental abnormality of the diaphragm musculature [1]. It usually remains asymptomatic in early life and presents later with respiratory and, occasionally, gastrointestinal complications. The abnormally wide sub-diaphragmatic space provides the potential for abnormal rotation of the stomach around itself. This abnormal rotation is known as gastric volvulus.

The most frequently used classification system for gastric volvulus, proposed by Singleton [2], relates to the axis around which the stomach rotates and is classified as follows:

Organo-axial - The stomach rotates around an axis that connects the gastrooesophageal junction and the pylorus.

The antrum rotates in the opposite direction to the fundus of the stomach.

This is the most common type of gastric volvulus, occurring in approximately $59 \%$ of cases [3], and is usually associated with diaphragmatic defects. Strangulation and necrosis commonly occur with this type and have been reported in 5-28\% of cases [4].

Mesentero-axial - The axis bisects the lesser and greater curvatures. The antrum rotates anteriorly and superiorly so that the posterior surface of the stomach lies anteriorly. The rotation is usually incomplete and occurs intermittently. Vascular compromise is uncommon. This aetiology comprises approximately $29 \%$ of cases [3].
Patients with this type usually present without diaphragmatic defects and usually have chronic symptoms.

Combined - This is a rare form in which the stomach twists mesentero-axially and organo-axially.

Combined gastric volvulus makes up the remainder of cases and is usually observed in patients with chronic volvulus [5].

According to the aetiology, gastric volvulus may be classified as either type 1 (idiopathic) or type 2 (congenital or acquired).

Type 1- This type comprises two thirds of cases and is presumably due to abnormal laxity of the gastro-splenic, gastroduodenal, gastro-phrenic, and gastrohepatic ligaments. This allows approximation of the cardia and pylorus when the stomach is full, predisposing to volvulus. Type 1 is more common in adults but has been reported in children.

Type 2 - This type is usually found in one third of patients and is associated with congenital or acquired abnormalities that result in abnormal mobility of the stomach, eg: diaphragmatic defects, asplenism and pyloric stenosis.

Gastric volvulus was first described by Berti in 1866 in a female autopsied patient, and the first operation was performed by Berg in 1897[6]. The clinical symptoms depend on the extent or degree of rotation and gastric outlet obstruction. Patients may present with the classical triad of Borchardt - epigastric distension, violent unproductive retching and inability to pass a nasogastric tube - our patient did not exhibit the last.

Patients with unilateral diaphragmatic eventeration are usually asymptomatic. However, some complain of dyspnoea on effort or rarely orthopnoea, due to the decrease in ventilation and oxygenation because of paradoxical motion of the affected diaphragm during inspiration and expiration. The severity of either symptom depends on the presence or absence of underlying pulmonary disease. 
The diagnosis of diaphragmatic eventeration associated with gastric volvulus is always difficult but awareness of the association should prompt the diagnosis which is established by history and, in most cases, by routine chest x-ray. Upper gastrointestinal endoscopy and computerized tomography with contrast are helpful.

Management of diaphragmatic eventeration varies greatly depending on whether the diagnosis is made in infants or adults. Simple cases may not require surgical intervention if it is not intruding significantly into the thoracic cavity and there are no adverse symptoms [1]. However, treatment by plication is indicated if there are symptoms of dyspnoea, recurrent pneumonia, chronic bronchitis, chest pain, poor exercise tolerance and functional disorders of the stomach[7]. Symptomatic gastric volvulus associated with diaphragmatic eventeration is a surgical emergency and requires surgical repair [8]. The most widely accepted approach is repair via an abdominal (subcostal) incision because this allows ready access to both hemi-diaphragms for plication, permits anterior gastric fixation via a gastropexy or gastrostomy, and allows abdominal exploration for associated gastrointestinal anomalies [8]. Recently, endoscopic reduction of the gastric volvulus has been reported [9] with reports of percutaneous endoscopic gastropexy (PEG) [10] and laparoscopic gastropexy [10] for gastric volvulus.

\section{References}

1. Wayne ER, Campbell JB, Burrington JD, Davis WS: Eventration of the diaphragm. J Pediatr Surg 1974; 9:643-651.
2. Singleton AC. Chronic gastric volvulus. Radiology. 1940; 34:53-61.

3. Milne LW, Hunter JJ, Anshus JS. Gastric volvulus: two cases and a review of the literature. J Emerg Med. 1994; 12:299-306.

4. Carter R, Brewer LA, Hinshaw DB. Acute gastric volvulus. A study of 25 cases. Am J Surg. 1980;140:99-106.

5. Wasselle JA, Norman J. Acute gastric volvulus: pathogenesis, diagnosis, and treatment. Am J Gastroenterol. 1993; $88: 1780-4$.

6. Berti A. singulare attortigliamento dele' esofago col duodeno seguita da rapida morte. Gazz Med Ital. 1866; 9:139.

7. Kizilcan F, Tanyel FC, Hiesomez A: The long term results of diaphragmatic plication. J Pediatr Surg 1993;28:42-4.

8. Par W, Choi S, Suh S: Paediatric gastric volvulus Experience with 7 cases. J Korean Med Sci. 1992; 7:258-263.

9. Tsang TK, Walker R, Yu DJ: Endoscopic reduction of gastric volvulus: the alphaloop maneuver. Gastrointest Endosc 1995; 42:244-8.

10. Baudet JS, Armengol-Mio JR, Medina C, Accarino AM, Vilaseca J, Malagelada JR:Percutaneous endoscopic gastrostomy as a treatment for chronic gastric volvulus. Endoscopy 1997; 29:147-148.

\section{Key Learning Points}

In gastric volvulus associated with diaphragmatic eventeration, organo-axial rotation is most common.

Mesentero-axial volvulus is intermittent and is not usually associated with vascular compromise. 